TRANSACTIONS OF THE

AMERICAN MATHEMATICAL SOCIETY

Volume 278, Number 1, July 1983

\title{
THE NEST SUBGROUPS OF KLEINIAN GROUPS
}

BY

TAKEHIKO SASAKI

\begin{abstract}
The residual limit points of finitely generated Kleinian groups are classified into two types: the first kind and the second kind. To each point of the second kind, Abikoff associated a web subgroup. We shall classify the points of the first kind into two types and associate to each point of one type a finitely generated subgroup, the nest subgroup. To the points of the other type we shall give a significance showing that they are important for the sets of generators.
\end{abstract}

Introduction. In his study of the residual limit sets of Kleinian groups, Abikoff, who is a pioneer in this direction, classified the residual limit points into two types, the first kind and the second kind, and then associated to each point of the second kind a subgroup and called it the web subgroup [1]. Later, Abikoff and Maskit [2] decomposed the finitely generated Kleinian groups into three kinds of subgroups: elementary, totally degenerate and web groups. The original groups may then be reconstructed from these groups by combination theorems. They also showed that web subgroups are web groups. Though the definition of the web groups is made by a property of component subgroups, in the case of the appearance of the residual limit points it is characterized by the property that the residual limit set consists of the points of the second kind only [3]. From the point of view of this characterization of the web groups, the nest groups are diametrically opposite, that is, the groups having the property that the residual limit set consists of the points of the first kind only [7]. In this article we shall associate to certain residual limit points of the first kind subgroups, which we shall call nest subgroups, as Abikoff did for web subgroups, and then show that nest subgroups are nest groups. The nest subgroups which we are going to define do not associate to all the residual limit points of the first kind, but to those which are characterized by separators of the special type. So we begin this article by classifying separators and then classifying the residual limit points into three types in $\S 1$. Under this classification of the residual limit points a theorem in [6] concerning the minimal sets of generators is refined in $\S 6$. In $\$ \S 2-5$ we define the nest subgroups and show they are nest groups.

I would like to express my gratitude to the referee for his informative advice.

1. Preliminaries and a new classification of the residual limit points. Let $G$ be a finitely generated Kleinian group, and denote by $\Omega(G)$ and $\Lambda(G)$ the region of discontinuity and the limit set of $G$, respectively. A component of $\Omega(G)$ is called a

Received by the editors March 25, 1982 and, in revised form, July 8, 1982.

1980 Mathematics Subject Classification. Primary 30F40.

Key words and phrases. Kleinian groups, web groups, nest groups, residual limit sets.

(C)1983 American Mathematical Society $0002-9947 / 82 / 0000-0770 / \$ 04.25$ 
component of $G$. A point $p$ of $\Lambda(G)$ is called a residual limit point if there is no component of $G$ on whose boundary $p$ lies. The set of all such points is called the residual limit set of $G$ and is denoted by $\Lambda_{0}(G)$. If $G$ is neither a function group nor a $Z_{2}$-extension of a quasi-Fuchsian group, then $\Lambda_{0}(G)$ is not empty $[\mathbf{1}, \mathbf{6}]$. Let $\Delta$ be a component of $G$. The component subgroup $G_{\Delta}$ is the stabilizer of $\Delta$ in $G$, and it is well known that $G_{\Delta}$ is finitely generated. The boundary of each component of $\hat{\mathbf{C}} \backslash\left(\Lambda\left(G_{\Delta}\right) \cup \Delta\right)$ is called a separator of $G$, where $\hat{\mathbf{C}}=\mathbf{C} \cup\{\infty\}$. Each separator of $G$ is a quasicircle contained in $\Lambda(G)$. Let $\sigma$ be a separator of $G$ and let $E_{1}$ and $E_{2}$ be sets of $\hat{\mathbf{C}}$ not contained in $\sigma$. We say that $\sigma$ separates $E_{1}$ from $E_{2}$ if $E_{1}$ lies in the closure of one component of $\hat{\mathbf{C}} \backslash \boldsymbol{\sigma}$ and $E_{2}$ lies in the closure of the other component of $\hat{\mathbf{C}} \backslash \sigma$. Let $\sigma$ be a separator lying on $\partial \Delta$, the boundary of $\Delta$, and let $E$ be an arbitrary set in $\hat{\mathbf{C}}$ separated from $\Delta$ by $\sigma$. Then the auxiliary domain of $\Delta$ relative to $E$ is the component of $\hat{\mathbf{C}} \backslash \sigma$ containing $\Delta$ and is denoted by $D(\Delta, E)$. The following are the fundamental properties of separators $[1, \mathbf{4}]$.

PROPOSITION 1.1. Separators do not cross each other.

Proposition 1.2. Separators form a null sequence with respect to the spherical metric.

A sequence of separators $\left\{\sigma_{n}\right\}$ is called a nest sequence if, for each $n, \sigma_{n}$ separates $\sigma_{n-1}$ from $\sigma_{n+1}$. Let $p$ be a point of $\Lambda_{0}(G)$. If there is a nest sequence of separators converging to $p$, then $p$ is called a point of the first kind. Otherwise, $p$ is called a point of the second kind. The sets of the residual limit points of the first and second kinds are denoted by $L_{1}(G)$ and $L_{2}(G)$, respectively.

Now we introduce a new classification of the residual limit points by the following classification of separators. It is shown in [5] that for each separator of $G$ there are at most two components of $G$ on whose boundaries it lies. So we classify the separators into two types: a separator $\sigma$ is of web type if $\sigma$ lies on the boundary of a single component of $G ; \sigma$ is of nest type if $\sigma$ lies on the boundaries of two components of $G$. Now, let $p$ be a point of $\Lambda_{0}(G)$. We say that $p$ is of web kind if there is no nest sequence of separators converging to $p ; p$ is of the nest kind if $p$ is not of the web kind and if there is no nest sequence of separators of web type converging to $p ; p$ is of the general kind if $p$ is neither of the web kind nor of the nest kind. We denote the sets of the residual limit points of the web, nest and general kinds by $L_{w}(G), L_{n}(G)$ and $L_{g}(G)$, respectively. The relation between the Abikoff and the new classifications is the following.

$$
L_{1}(G)=L_{n}(G)+L_{g}(G) \text { and } L_{2}(G)=L_{w}(G) .
$$

A finitely generated Kleinian group is called a web group if each component subgroup is quasi-Fuchsian [2]. The web subgroup $W=W(p)$ of a residual limit point of the second kind $p$ is a subgroup of $G$ constructed as follows [1]: Let $q \in \Omega(G)$ and let $S(q, p)$ be the set of all the separators of $G$ which separate $p$ from $q$. Then there exists a separator $s(q, p)$ in $S(q, p)$ such that there is no separator in $S(q, p)$, except for $s(q, p)$, which lies in the closure of the component of $\hat{\mathbf{C}} \backslash s(q, p)$ containing $p$. This separator is called a maximal separator and we set $\mathfrak{M}(p)=$ $\{s(q, p) \mid q \in \Omega(G)\}$. Then $W$ is the stabilizer of $\Phi(p)$ in $G$, where $\Phi(p)$ is the 
closure of the set $\mathfrak{M}(p)$ and is called the web of $p$. It is shown in [2] that the web subgroup of each point of $L_{2}(G)$ is a web group. A finitely generated Kleinian group with the nonempty residual limit set is called a nest group if each residual limit point is of the first kind [7]. By our classifications of separators and residual limit points, a characterization of these two groups is given as follows.

Proposition 1.3. Let $G$ be a finitely generated Kleinian group with $\Lambda_{0}(G) \neq \varnothing$. Then (i)-(iii) are equivalent.

(i) $G$ is a web group;

(ii) $\Lambda_{0}(G)=L_{w}(G)$; and

(iii) $L_{g}(G)=\varnothing$ and each separator of $G$ is of web type.

Further (iv)-(vi) are equivalent.

(iv) $G$ is a nest group;

(v) $\Lambda_{0}(G)=L_{n}(G)$; and

(vi) each separator of $G$ is of nest type.

Proof. (i) $\Leftrightarrow$ (ii) is shown in [3].

(i) and (ii) $\Rightarrow$ (iii). Since $\Lambda_{0}(G)=L_{w}(G)$, then $L_{g}(G)=\varnothing$. If there is a separator of nest type $\sigma$, then both components of $\hat{\mathbf{C}} \backslash \sigma$ must be components of $G$ by (i). Hence $\Lambda(G)=\sigma$. This contradicts the assumption that $\Lambda_{0}(G) \neq \varnothing$. Hence each separator of $G$ is of web type. Thus we have (iii).

(iii) $\Rightarrow$ (ii). Since $L_{g}(G)=\varnothing$, we have $\Lambda_{0}(G)=L_{n}(G)+L_{w}(G)$. Since each separator of $G$ is of web type, there is no nest sequence of separators of nest type. Hence $L_{n}(G)=\varnothing$. Thus we have (ii).

The equivalence of (iv) and (vi) is shown in [7]. It is clear from the definition of nest groups that $(\mathrm{v}) \Rightarrow$ (iv).

Lastly we show that (iv) and (vi) $\Rightarrow(\mathrm{v})$. Since (iv) $\Rightarrow L_{w}(G)=\varnothing$ and (vi) $\Rightarrow L_{g}(G)$ $=\varnothing, \Lambda_{0}(G)=L_{n}(G)$. Hence we have (v). Q.E.D.

The following gives us a reason why we use the terminology of web type.

LEMMA 1.4. Let $G$ be a finitely generated Kleinian group with $\Lambda_{0}(G) \neq \varnothing$. If there is a separator of web type $\sigma$, then there is a point $p$ of $L_{w}(G)$ such that $\sigma$ is a separator of the web subgroup $W(p)$.

Proof. Lemma 1.4 is proved implicitly in the proof of Theorem 4 in [7]. For the sake of completeness we outline it. Let $\Delta$ be the component of $G$ on whose boundary $\sigma$ lies. Let $\left\{\sigma_{k}\right\}$ be a list of the separators for $G$ not lying in $\partial \Delta$ and not separated from $\sigma$ by another separator. Clearly $\Phi=\overline{\cup_{k} \sigma_{k}}$ is a web of some point $p \in L_{w}(G)$ and $\sigma$ is a separator for $W(p)=G_{\Phi}$.

The following is implicit in Abikoff [1].

Proposition 1.5. Let $W=W(p)$ be the web subgroup of $p, p \in L_{w}(G)$. Then

(i) $\Lambda_{0}(W) \subset L_{w}(G)$ and

(ii) each separator of $W$ is a separator of web type of $G$.

2. Nest $\Psi(p)$. Let $G$ be a finitely generated Kleinian group with $L_{n}(G) \neq \varnothing$. Assuming that $G$ is not a nest group, we shall associate to each point of $L_{n}(G)$ a 
proper subgroup of $G$. As the first step of this we define an invariant set $\Psi(p)$ of the subgroup.

Let $p \in L_{n}(G)$. By our assumption that $\Lambda_{0}(G) \supset L_{n}(G) \neq \varnothing$, we see by Proposition 1.3 that $L_{w}(G) \neq \varnothing$. Let $q$ be a point of $L_{w}(G)$. Then there are separators of web type separating $p$ from $q$. For example, the boundary of the component of the web subgroup of $q$ containing $p$ is such a separator. By Proposition 1.2 and by the choice of $p$ and $q$, the number of such separators is finite. We denote by $\sigma(q, p)$ the nearest one to $p$, that is, $\sigma(q, p)$ is a separator of web type separating $p$ from $q$ such that the component of $\hat{\mathbf{C}} \backslash \sigma(q, p)$ containing $p$ does not intersect any separator of web type separating $p$ from $q$. We put $M(p)=\left\{\sigma(q, p) \mid q \in L_{w}(G)\right\}$ and define $\Psi(p)$ to be the closure of the set $M(p)$. We shall call $\Psi(p)$ the nest of $p$.

REMARK. In general, to a point $q$ of $\Omega(G)$ there does not correspond $\sigma(q, p)$. Hence in the construction of $M(p)$ we must restrict $q$ to be in $L_{w}(G)$. The validity of a name, the nest of $p$, will be given by Theorems 3.5 and 5.4 .

Now we shall investigate some properties of nests.

Proposition 2.1. $p \in \Psi(p)$.

Proof. Since $L_{w}(G)$ is an invariant set of the nonelementary discrete group $G$, there is a sequence $\left\{q_{n}\right\}$ of points of $L_{w}(G)$ converging to $p$. By Proposition 1.2 the sequence $\left\{\sigma\left(q_{n}, p\right)\right\}$ of the separators of $M(p)$ converges to $p$ so $p \in \Psi(p)$.

Proposition 2.2. Let $p_{1}$ and $p_{2}$ be points of $L_{n}(G)$. Then $\Psi\left(p_{1}\right)=\Psi\left(p_{2}\right)$ iff there is no separator of web type separating $p_{1}$ from $p_{2}$.

Proof. Necessity. Assume there is a separator of web type $\sigma$ separating $p_{1}$ from $p_{2}$. Then $M\left(p_{1}\right)$ (and hence also $\Psi\left(p_{1}\right)$ ) lies in the closure of the component of $\hat{\mathbf{C}} \backslash \sigma$ containing $p_{1}$. On the other hand, $p_{2}$ lies in the component $\hat{\mathbf{C}} \backslash \sigma$ not containing $p_{1}$, so $p_{2} \notin \Psi\left(p_{1}\right)$. Hence we have by Proposition 2.1 that $\Psi\left(p_{1}\right) \neq \Psi\left(p_{2}\right)$.

Sufficiency. Let $q \in L_{w}(G)$. Since $\sigma\left(q, p_{1}\right)$ is of web type, it does not separate $p_{1}$ from $p_{2}$, so $\sigma\left(q, p_{1}\right)$ separates $p_{2}$ from $q$. We assert that $\sigma\left(q, p_{1}\right) \in M\left(p_{2}\right)$. If $\sigma\left(q, p_{1}\right) \neq \sigma\left(q, p_{2}\right)$, then $\sigma\left(q, p_{2}\right)$ must lie in the closure of the component of $\hat{\mathbf{C}} \backslash \sigma\left(q, p_{1}\right)$ containing $p_{1}$. Since $\sigma\left(q, p_{2}\right)$ is of web type, it does not separate $p_{1}$ from $p_{2}$. Hence $\sigma\left(q, p_{2}\right)$ separates $p_{1}$ from $\sigma\left(q, p_{1}\right)$. This contradicts that $\sigma\left(q, p_{1}\right) \in$ $M\left(p_{1}\right)$. Thus we have our assertion that $\sigma\left(q, p_{1}\right) \in M\left(p_{2}\right)$ so $M\left(p_{1}\right) \subset M\left(p_{2}\right)$. Similarly, $M\left(p_{2}\right) \subset M\left(p_{1}\right)$ so $M\left(p_{1}\right)=M\left(p_{2}\right)$. Hence $\Psi\left(p_{1}\right)=\Psi\left(p_{2}\right)$. Q.E.D.

We denote by $c(q, p)$ the component of $\hat{\mathbf{C}} \backslash \sigma(q, p)$ containing $q$ and put $C(p)=$ $\left\{c(q ; p) \mid q \in L_{w}(G)\right\}$.

Proposition 2.3. $c(q, p)$ is a component of $\hat{\mathbf{C}} \backslash \Psi(p)$.

Proof. Since each point of $c(q, p)$ is separated from $p$ by $\sigma(q, p)$, there is no separator of $M(p)$ lying in the closure of $c(q, p)$ other than $\sigma(q, p)$ so $c(q, p)$ does not contain any point of $\Psi(p)$. Since $\sigma(q, p)$ is the boundary of $c(q, p)$ and is contained in $\Psi(p), c(q, p)$ is a component of $\hat{\mathbf{C}} \backslash \Psi(p)$. Q.E.D.

In the rest of this section we observe some relations between the components of $G$ and the elements of $C(p)$. 
Proposition 2.4. Let $\Delta$ be a component of $G$. If $\Delta$ is contained in an element of $C(p)$, then $\Delta$ is not a component of $\hat{\mathbf{C}} \backslash \Psi(p)$.

Proof. Let $\Delta \subset c(q, p)$. Since $q \in c(q, p)$ and is a limit point of $G, \Delta \underset{\neq}{\subset} c(q, p)$. Proposition 2.3 implies that $\Delta$ is not a component of $\hat{\mathbf{C}} \backslash \Psi(p)$.

Proposition 2.5. Let $\Delta$ be a component of $G$. Then $\Delta$ is contained in an element of $C(p)$ iff there is a separator of web type separating $p$ from $\Delta$.

Proof. Sufficiency. Let $\sigma$ be a separator of web type separating $p$ from $\Delta$. Let $D_{\Delta}$ and $D_{p}$ be the components of $\hat{\mathbf{C}} \backslash \sigma$ containing $\Delta$ and $p$, respectively. If $D_{\Delta} \cap L_{w}(G)$ $\neq \varnothing$, let $q$ be a point of $D_{\Delta} \cap L_{w}(G)$. Then either $\sigma(q, p)=\sigma$ or $\sigma(q, p)$ separates $p$ from $\sigma$. Hence $\Delta \subset c(q, p)$. If $D_{\Delta} \cap L_{w}(G)=\varnothing$, let $W=W(q)$ be the web subgroup in Lemma 1.4 having $\sigma$ as a separator. Then $q \in D_{p} \cap L_{w}(G)$. Since there is no separator separating $q$ from $\sigma, \sigma(q, p)$ does not separate $q$ from $\sigma$, so that it separates $p$ from $\Delta$. Hence $\Delta \subset c(q, p)$.

Conversely, if $\Delta \subset c(q, p)$, then $\sigma(q, p)$ is a separator of web type separating $p$ from $\Delta$.

COROLlaRY 2.6. Let $\Delta_{1}$ and $\Delta_{2}$ be components of $G$. If $\Delta_{1}$ and $\Delta_{2}$ are not contained in elements of $C(p)$, then there is no separator of web type separating $\Delta_{1}$ from $\Delta_{2}$.

Proof. Assume there is a separator of web type $\sigma$ separating $\Delta_{1}$ from $\Delta_{2}$. Since $p$ does not lie on $\sigma$, $\sigma$ separates $p$ from either $\Delta_{1}$ or $\Delta_{2}$. This is impossible by Proposition 2.5.

3. Nest subgroup $N(p)$. We shall call the stabilizer of $\Psi(p)$ in $G$ the nest subgroup of $p$ and denote it by $N(p)$. Our goal of this section is to show that $\Lambda(N(p))=\Psi(p)$.

LEMMA 3.1. Let $\Delta$ be a component of $G$. If $\Delta$ is not contained in any element of $C(p)$, then $G_{\Delta}$ is a subgroup of $N(p)$.

Proof. Proposition 2.5 implies there is no separator of web type separating $p$ from $\Delta$. Hence there is no separator of web type separating $g(p)$ from $g(\Delta)=\Delta, g \in G_{\Delta}$. Therefore there is no separator of web type separating $p$ from $g(p)$. Then Proposition 2.2 implies $\Psi(p)=\Psi(g(p))$. Now, if $\sigma \in M(p)$, then $g(\sigma) \in M(g(p)) \subset$ $\Psi(g(p))=\Psi(p)$. Thus $g(M(p)) \subset \Psi(p)$ so $g(\Psi(p)) \subset \Psi(p)$ for each $g \in G_{\Delta}$. Hence $g(\Psi(p)) \subset \Psi(p)=g\left(g^{-1}(\Psi(p))\right) \subset g(\Psi(p))$ or $g(\Psi(p))=\Psi(p)$, by which we have $G_{\Delta} \subset N(p)$. Q.E.D.

COROllary 3.2. A component of $G$ is a component of $N(p)$ iff it is not contained in any element of $C(p)$.

Proof. Let $\Delta$ be a component of $G$ not contained in any element of $C(p)$. Then Lemma 3.1 implies that $G_{\Delta}$ is a subgroup of $N(p)$. Therefore $\partial \Delta=\Lambda\left(G_{\Delta}\right) \subset$ $\Lambda(N(p))$. Since $\Delta$ is a component of $G, \Delta$ contains no limit point of any subgroup of $G$ so $\Delta \cap \Lambda(N(p))=\varnothing$. Hence $\Delta$ is a component of $N(p)$.

Conversely, let $\Delta$ be a component of both $G$ and $N(p)$. Assume that $\Delta$ is contained in an element $c(q, p)$ of $C(p)$. Then, since $q \in c(q, p) \cap \Lambda(G)$, 
$\Delta \subset c(q, p)$. On the other hand, since $\Lambda(N(p)) \subset \Psi(p)$, each component of $\hat{\mathbf{C}} \backslash \Psi(p)$ is contained in a component of $N(p)$, so by Proposition 2.3, $c(q, p) \subset \Delta$, a contradiction. Hence $\Delta$ is not contained in any element of $C(p)$.

Proposition 3.3. The component of $G$ on whose boundary $\sigma(q, p)$ lies is a component of $N(p)$.

Proof. Let $\Delta$ be the component of $G$ such that $\sigma(q, p) \subset \partial \Delta$. By Corollary 3.2 we need only show that $\Delta$ is not contained in any element of $C(p)$. We first show that $\Delta$ lies outside $c(q, p)$. Let $W$ be the web subgroup in Lemma 1.4 having $\sigma(q, p)$ as a separator. Let $\Delta^{*}$ be the component of $W$ containing $\Delta$. Since $\sigma(q, p) \subset \partial \Delta$, $\partial \Delta^{*}=\sigma(q, p)$. If $\Delta \subset c(q, p)$, then $c(q, p)=\Delta^{*}$. Hence $p$ lies outside $\Delta^{*}$. Since $p \in L_{n}(G)$, by Proposition 1.5, $p \in \Omega(W)$. Let $\Delta_{p}^{*}$ be the component of $W$ containing $p$. Then $\Delta_{p}^{*} \neq \Delta^{*}$ and $\partial \Delta_{p}^{*}$ separates $p$ from $\sigma(q, p)$. Since $\partial \Delta_{p}^{*}$ is of web type by Proposition 1.5, $\sigma(q, p) \notin M(p)$, a contradiction. Thus $\Delta$ lies outside $c(q, p)$.

Next, assuming $c\left(q^{\prime}, p\right) \neq c(q, p)$, we show that $\Delta$ lies outside $c\left(q^{\prime}, p\right)$. If $\Delta$ is contained in $c\left(q^{\prime}, p\right)$, then $\partial \Delta$ is contained in the closure of $c\left(q^{\prime}, p\right)$. Then $\sigma(q, p)$ is contained in the closure of both $c(q, p)$ and $c\left(q^{\prime}, p\right)$. Since $c(q, p)$ and $c\left(q^{\prime}, p\right)$ are disjoint by Proposition 2.3, $c(q, p) \cup c\left(q^{\prime}, p\right) \cup \sigma(q, p)=\hat{\mathbf{C}}$. Since $p$ does not lie in $c(q, p), c\left(q^{\prime}, p\right)$ or on $\sigma(q, p)$, this is impossible. Therefore $\Delta$ lies outside $c\left(q^{\prime}, p\right)$. Hence there is no element of $C(p)$ containing $\Delta$. Q.E.D.

Proposition 3.4. $c(q, p)$ is a component of $N(p)$.

Proof. Let $\Delta$ be the component of $G$ on whose boundary $\sigma(q, p)$ lies. Proposition 3.3 implies that $\Delta$ is a component of $N(p)$. Corollary 3.2 implies that $\Delta$ is not contained in any element of $C(p)$. Lemma 3.1 implies that $\partial c(q, p)=\sigma(q, p) \subset \partial \Delta$ $=\Lambda\left(G_{\Delta}\right) \subset \Lambda(N(p))$. By Proposition 2.3, $c(q, p) \cap \Lambda(N(p)) \subset c(q, p) \cap \Psi(p)=$ $\varnothing$. Hence $c(q, p)$ is a component of $N(p)$.

THEOREM 3.5. $\Lambda(N(p))=\Psi(p)$.

Proof. Proposition 3.4 implies that $\sigma(q, p)$ lies in $\Lambda(N(p))$. Hence $M(p) \subset$ $\Lambda(N(p))$ so $\Psi(p) \subset \Lambda(N(p))$. The inverse inclusion is clear. Q.E.D.

4. Linkage of components $\mathcal{L}(\Delta)$. In our proof that $N(p)$ is finitely generated, linkages of components will play a fundamental role. In this section we define linkages of components and investigate some properties of them.

Let $\Delta$ and $\Delta^{\prime}$ be distinct components of $G$. We shall say that $\Delta^{\prime}$ is linked to $\Delta$ by separators of nest type if there is a finite set of distinct separators of nest type $\left\{\sigma_{i}\right\}_{i=1}^{n}$ such that $\sigma_{1}=\partial D\left(\Delta, \Delta^{\prime}\right), \sigma_{n}=\partial D\left(\Delta^{\prime}, \Delta\right)$, there is a component of $G$ on whose boundary $\sigma_{i}$ and $\sigma_{i+1}$ lie (if $n>1$ ), and there is no component of $G$ on whose boundary $\sigma_{i}$ and $\sigma_{i+2}$ lie (if $n>2$ ), $i=1,2, \ldots, n-2$. We call $n$ the length of the linkage between $\Delta$ and $\Delta^{\prime}$. Clearly the relation that two components are linked by separators of nest type is an equivalence one. We shall denote by $\mathcal{L}(\Delta)$ this equivalence class of components containing $\Delta$. Clearly we have the following.

Proposition 4.1. If $\Delta^{\prime} \in \mathcal{L}(\Delta)$, then $\mathcal{L}\left(\Delta^{\prime}\right)=\mathcal{L}(\Delta)$. 
Proposition 4.2. If there is a component $\Delta$ of both $G$ and $N(p)$, then $\mathcal{L}(\Delta)$ is the set of all components of $N(p)$ not contained in any element of $C(p)$.

Proof. Corollary 3.2 implies that $\Delta$ is not contained in any element of $C(p)$. Let $\Delta^{\prime}$ be an element of $\mathcal{L}(\Delta)$. Then $\Delta^{\prime}$ is linked to $\Delta$ by separators of nest type, hence there is no separator of web type separating $\Delta$ from $\Delta^{\prime}$. Proposition 2.5 implies there is no separator of web type separating $p$ from $\Delta$. Hence there is no separator of web type separating $p$ from $\Delta^{\prime}$. Therefore, by Proposition $2.5, \Delta^{\prime}$ is not an element of $C(p)$. Corollary 3.2 implies that $\Delta^{\prime}$ is a component of $N(p)$. Thus each element of $\mathcal{L}(\Delta)$ is a component of $N(p)$ not contained in any element of $C(p)$.

Next we show that each component of $N(p)$ not contained in any element of $C(p)$ is an element of $\mathcal{L}(\Delta)$. Let $\Delta^{\prime}$ be a component of $N(p)$ not contained in any element of $C(p)$. Then Corollary 3.2 implies that $\Delta^{\prime}$ is a component of $G$. Corollary 2.6 implies that there is no separator of web type separating $\Delta$ from $\Delta^{\prime}$. Proposition 1.2 implies that there are only a finite number of separators of nest type separating $\Delta$ from $\Delta^{\prime}$, hence $\Delta^{\prime}$ is linked to $\Delta$ by them. Therefore $\Delta^{\prime} \in \mathcal{L}(\Delta)$. Q.E.D.

THEOREM 4.3. There is a component $\Delta$ of $G$ such that the set of all components of $N(p)$ is the disjoint union of $\mathcal{L}(\Delta)$ and $C(p)$.

Proof. Proposition 3.4 implies that each element of $C(p)$ is a component of $N(p)$. Proposition 3.3 implies that there exists a component of both $G$ and $N(p)$. Let $\Delta$ be such a component. Then Proposition 4.2 implies that $\mathcal{L}(\Delta)$ is the set of all components of $N(p)$ which are not elements of $C(p)$. Q.E.D.

CoRollary 4.4. Let $\Delta$ be as in Theorem 4.3. Then for each $c(q, p)$ there is an element $\Delta^{\prime}$ of $\mathcal{L}(\Delta)$ such that $c(q, p)$ is a component of $G_{\Delta^{\prime}}$.

Proof. Let $\Delta^{\prime}$ be the component in Proposition 3.3 such that $\Delta^{\prime}$ is a component of both $N(p)$ and $G$ and $\sigma(q, p) \subset \partial \Delta^{\prime}$. Corollary 3.2 and Theorem 4.3 imply $\Delta^{\prime} \in \mathcal{L}(\Delta)$. By Theorem 4.3, $\Delta^{\prime} \cap c(q, p)=\varnothing$. Hence $\partial c(q, p) \cap \partial \Delta^{\prime}=\sigma(q, p)$. Since $\Lambda\left(G_{\Delta^{\prime}}\right)$ $=\partial \Delta^{\prime}, c(q, p)$ is a component of $G_{\Delta^{\prime}}$.

LEMMA 4.5. Let $g$ be an element of $G$. If there is a component $\Delta$ of $N(p)$ such that $g(\Delta)$ is a component of $N(p)$, then $g \in N(p)$.

Proof. We first show that we may assume $\Delta$ is a component of $G$ so both $\Delta$ and $g(\Delta)$ belong to $\mathcal{L}(\Delta)$. If $\Delta$ is not a component of $G$, then there is an element $c(q, p)$ of $C(p)$ such that $\Delta=c(q, p)$. Then Corollary 4.4 implies that there is a component $\Delta^{\prime}$ of $\mathcal{E}\left(\Delta^{\prime \prime}\right)$ with $\partial \Delta \cap \partial \Delta^{\prime}=\sigma(q, p)$, where $\Delta^{\prime \prime}$ is the component in Theorem 4.3. Since $g(\Delta)$ is a component of $N(p)$ but not of $G$, by Theorem $4.3 g(\Delta)$ is an element of $C(p)$ so $g(\Delta)=c\left(q^{\prime}, p\right)$ for some $q^{\prime} \in L_{w}(G)$. Since $g\left(\Delta^{\prime}\right)$ is a component of $G$, and since $\partial g\left(\Delta^{\prime}\right)=g\left(\partial \Delta^{\prime}\right) \supset g(\sigma(q, p))=\sigma\left(q^{\prime}, p\right)$, Proposition 3.3 implies that $g\left(\Delta^{\prime}\right)$ is a component of $N(p)$. Clearly $\Delta^{\prime}$ is a component of $N(p)$. Therefore, replacing $\Delta$ by $\Delta^{\prime}$, we may assume that $\Delta$ is a component of $G$ so both $\Delta$ and $g(\Delta)$ belong to $\mathcal{L}(\Delta)$.

Let $\Delta^{\prime}$ be an element of $\mathcal{L}(\Delta)$ and let $\left\{\sigma_{i}\right\}_{i=1}^{n}$ be the linkage of separators of nest type from $\Delta$ to $\Delta^{\prime}$. Then $\left\{g\left(\sigma_{i}\right)\right\}_{i=1}^{n}$ is the linkage of the separators of nest type from 
$g(\Delta)$ to $g\left(\Delta^{\prime}\right)$. Hence $g\left(\Delta^{\prime}\right) \in \mathcal{L}(g(\Delta))=\mathcal{L}(\Delta)$ so $g(\mathcal{L}(\Delta)) \subset \mathcal{L}(\Delta)$. Since both $g(\Delta)$ and $g^{-1}(g(\Delta))$ belong to $\mathcal{L}(\Delta)$, we have, similarly, $g^{-1}(\mathcal{L}(\Delta)) \subset \mathcal{L}(\Delta)$. Therefore,

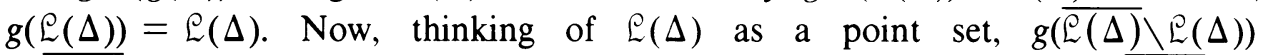
$=\overline{\mathscr{L}(\Delta)} \backslash \mathscr{L}(\Delta)$. Since $\mathscr{L}(\Delta)$ is an invariant set of $N(p), \Lambda(N(p)) \subset \overline{\mathcal{L}(\Delta)}$ so $\Lambda(N(p)) \subset \overline{\mathcal{L}(\Delta)} \backslash \Omega(N(p)) \subset \Lambda(N(p))$ or $\Lambda(N(p))=\overline{\mathcal{L}(\Delta)} \backslash \Omega(N(p))$. Since $\overline{\mathcal{L}(\Delta)}$ $\cap C(p)=\varnothing, \Lambda(N(p))=\overline{\mathcal{L}(\Delta)} \backslash \mathcal{L}(\Delta)$. Hence by Theorem 3.5,

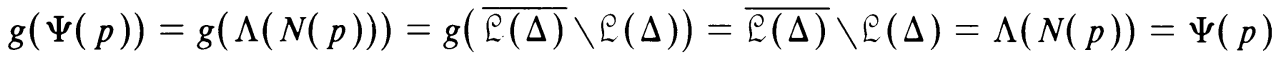
so $g \in N(p)$. Q.E.D.

COROLlaRY 4.6. The equivalence of components of $\mathcal{L}(\Delta)$ in $G$ is identical with the equivalence in $N(p)$, where $\Delta$ is in Theorem 4.3.

Proof. Let $\Delta_{1}$ and $\Delta_{2}$ be elements of $\mathcal{L}(\Delta)$. If $g\left(\Delta_{1}\right)=\Delta_{2}$ with $g \in G$, then $g\left(\Delta_{1}\right)=\Delta_{2} \in \mathcal{L}(\Delta)=\mathcal{L}\left(\Delta_{1}\right)$. Lemma 4.5 implies that $g \in N(p)$. Clearly the equivalence of $\Delta_{1}$ and $\Delta_{2}$ in $N(p)$ implies their equivalence in $G$. Q.E.D.

Though the next lemma is used in $\S 6$, it seems appropriate to prove it here.

LEMMA 4.7. Let $g$ be a loxodromic element of $G$ with fixed points $\xi_{1}$ and $\xi_{2}$ on $L_{n}(G)$. Then $g \in N\left(\xi_{1}\right)=N\left(\xi_{2}\right)$.

Proof. If there is a separator of web type $\sigma$ separating $\xi_{1}$ from $\xi_{2}$, then the sequence of separators of web type $\left\{g^{n}(\sigma)\right\}_{n=1}^{\infty}$ converges to either $\xi_{1}$ or $\xi_{2}$, say $\xi_{1}$. Then $\xi_{1} \in L_{g}(G)$ so $\xi_{1} \notin L_{n}(G)$. Hence there is no separator of web type separating $\xi_{1}$ from $\xi_{2}$. Then Proposition 2.2 implies that $\Psi\left(\xi_{1}\right)=\Psi\left(\xi_{2}\right)$ so $N\left(\xi_{1}\right)=N\left(\xi_{2}\right)$.

Next we show $g \in N\left(\xi_{1}\right)$. Let $\Delta$ be a component of both $G$ and $N\left(\xi_{1}\right)$. By Lemma 4.5 we need only show that $g(\Delta)$ is a component of $N\left(\xi_{1}\right)$. By Theorem 4.3 it suffices to show that $g(\Delta) \in \mathcal{L}(\Delta)$. If $g(\Delta) \notin \mathcal{L}(\Delta)$, then there is an element $c\left(q, \xi_{1}\right)$ of $C\left(\xi_{1}\right)$ such that $g(\Delta) \subset c\left(q, \xi_{1}\right)$. Since $\sigma\left(q, \xi_{1}\right)$ separates $\xi_{1}$ from $g(\Delta), g^{-1}\left(\sigma\left(q, \xi_{1}\right)\right)$ separates $\xi_{1}$ from $\Delta$. Proposition 2.5 implies that $\Delta$ is contained in an element of $C\left(\xi_{1}\right)$. This contradicts Corollary 3.2. Hence $g(\Delta) \in \mathcal{L}(\Delta)$. Q.E.D.

5. $N(p)$ is a nest group. The purpose of this section is to show that $N(p)$ is a nest group, in particular that it is finitely generated.

PROPOSITION 5.1. There are only a finite number of inequivalent components of $N(p)$.

Proof. The Ahlfors finiteness theorem tells us there is only a finite number of $G$-inequivalent components of $G$. By Corollary 4.6 there is only a finite number of $N(p)$-inequivalent elements in $\mathcal{L}(\Delta)$. Corollary 4.4 implies that each element of $C(p)$ is a component of some component subgroup $G_{\Delta^{\prime}}, \Delta^{\prime} \in \mathcal{L}(\Delta)$. Since there is only a finite number of $G_{\Delta^{\prime}}$-inequivalent components of $G_{\Delta^{\prime}}$, and $G_{\Delta^{\prime}}$ is a subgroup of $N(p)$ by Lemma 3.1, there is only a finite number of $N(p)$-inequivalent components of $G_{\Delta^{\prime}}$. Since there is only a finite number of $N(p)$-inequivalent elements in $\mathcal{L}(\Delta)$, there is only a finite number of nonconjugate $G_{\Delta^{\prime}}$ in $N(p)$. Therefore there is only a finite number of $N(p)$-inequivalent elements in $C(p)$. By Theorem 4.3 we have Proposition 5.1. Q.E.D. 
For each positive integer $i$ we denote by $E_{i}(\Delta)$ the subset of $\mathcal{L}(\Delta)$ consisting of all elements whose lengths of linkage from $\Delta$ are at most $i$. Let $N_{i}(\Delta)$ be the subgroup of $N(p)$ generated by all component subgroups $G_{\Delta^{\prime}}, \Delta^{\prime} \in \mathcal{L}_{i}(\Delta)$.

LEMmA 5.2. There is a finite subset $\left\{\Delta_{1}, \ldots, \Delta_{n_{1}}\right\}$ of $\varrho_{i}(\Delta)$ such that $N_{i}(\Delta)=$ $\left\langle G_{\Delta_{1}}, \ldots, G_{\Delta_{n}}\right\rangle$ and each element of $\mathcal{L}_{i}(\Delta)$ is $N_{i}(\Delta)$-equivalent to some element of $\left\{\Delta_{1}, \ldots, \Delta_{n_{i}}\right\}$. In particular, $N_{i}(\Delta)$ is finitely generated $(i=1,2, \ldots)$.

Proof. Since there is only a finite number of $G_{\Delta}$-inequivalent separators on $\partial \Delta$, there is only a finite number of $G_{\Delta}$-inequivalent elements in $\varrho_{1}(\Delta)$. Let them be $\left\{\Delta_{1}, \ldots, \Delta_{n_{1}}\right\}$ with $\Delta_{1}=\Delta$. Then $N_{1}(\Delta)=\left\langle G_{\Delta_{1}}, \ldots, G_{\Delta_{n_{1}}}\right\rangle$ and each element of $\mathcal{E}_{1}(\Delta)$ is $N_{1}(\Delta)$-equivalent to some element of $\left\{\Delta_{1}, \ldots, \Delta_{n_{1}}\right\}$. Hence the lemma is true for $i=1$. Assuming the lemma is true for $i$, we show it is true for $i+1$. Let $\Delta^{\prime}$ be an element of $\mathcal{L}_{i+1}(\Delta) \backslash E_{i}(\Delta)$. Then there is an element $\Delta^{\prime \prime}$ of $\mathcal{L}_{i}(\Delta)$ whose length of the linkage to $\Delta^{\prime}$ is 1 . Since $\Delta^{\prime \prime}$ is $N_{i}(\Delta)$-equivalent to some component $\Delta_{j}$ of $\left\{\Delta_{1}, \ldots, \Delta_{n}\right\}$, there is an element $g$ of $N_{i}(\Delta)$ such that $\Delta^{\prime \prime}=g\left(\Delta_{j}\right)$. Then each element of $\mathcal{L}_{1}\left(\Delta^{\prime \prime}\right)$ is a $g$-image of an element of $\mathcal{L}_{1}\left(\Delta_{j}\right)$ so that $N_{1}\left(\Delta^{\prime \prime}\right)=g N_{1}\left(\Delta_{j}\right) g^{-1}$. Hence

$$
\begin{aligned}
G_{\Delta^{\prime}} & \subset N_{1}\left(\Delta^{\prime \prime}\right) \subset\left\langle g, g^{-1} N_{1}\left(\Delta^{\prime \prime}\right) g\right\rangle \subset\left\langle N_{i}(\Delta), N_{1}\left(\Delta_{j}\right)\right\rangle \\
& \subset\left\langle N_{i}(\Delta), N_{1}\left(\Delta_{1}\right), \ldots, N_{1}\left(\Delta_{n_{1}}\right)\right\rangle
\end{aligned}
$$

so

$$
N_{i+1}(\Delta) \subset\left\langle N_{i}(\Delta), N_{1}\left(\Delta_{1}\right), \ldots, N_{1}\left(\Delta_{n_{i}}\right)\right\rangle .
$$

In order to show the inverse inclusion, let $\left\{\Delta_{1}^{*}, \ldots, \Delta_{m_{j}}^{*}\right\}$ be a subset of $\varrho_{1}\left(\Delta_{j}\right)$ such that $N_{1}\left(\Delta_{j}\right)=\left\langle G_{\Delta_{i}^{*}}, \ldots, G_{\Delta_{m_{j}}^{*}}\right\rangle\left(j=1,2, \ldots, n_{i}\right)$. Since $\Delta_{k}^{*} \in \mathcal{L}(\Delta)\left(k=1, \ldots, m_{j}\right)$ and the length of the linkage from $\Delta$ to $\Delta_{k}^{*}$ is at most $i+1, \Delta_{k}^{*} \in \mathcal{L}_{i+1}(\Delta)$. Hence $G_{\Delta_{k}^{*}} \subset N_{i+1}(\Delta)$ so $N_{1}\left(\Delta_{j}\right) \subset N_{i+1}(\Delta)\left(j=1, \ldots, n_{i}\right)$. Since $N_{i}(\Delta) \subset N_{i+1}(\Delta)$, we have the inverse inclusion so

$$
N_{i+1}(\Delta)=\left\langle N_{i}(\Delta), N_{1}\left(\Delta_{1}\right), \ldots, N_{1}\left(\Delta_{n_{i}}\right)\right\rangle .
$$

By our induction hypothesis $N_{i+1}(\Delta)$ is finitely generated so there is a finite subset $\left\{\Delta_{1}, \ldots, \Delta_{n_{i+1}}\right\}$ of $\mathcal{L}_{i+1}(\Delta)$ having the desired properties. Hence the lemma is true for $i+1$. Q.E.D.

Lemma 5.2 implies that each element of $\varrho_{i}(\Delta)$ is $N_{i}(\Delta)$-equivalent to an element of $\left\{\Delta_{1}, \ldots, \Delta_{n_{i}}\right\} \subset \mathscr{L}_{i}(\Delta)$. Then each element of $\mathcal{L}_{i}(\Delta)$ is $N(p)$-equivalent to an element of a subset of $\left\{\Delta_{1}, \ldots, \Delta_{n_{i}}\right\}$. Hence, adding a finite number of elements of $N(p)$ to a finite set of generators of $N_{i}(\Delta)$, we obtain a group $N_{i}^{*}(\Delta)$ with the following properties.

(a) $N_{i}^{*}(\Delta)$ is a finitely generated subgroup of $N(p)$, and

(b) if two elements of $\mathcal{L}_{i}(\Delta)$ are $N(p)$-equivalent, then they are $N_{i}^{*}(\Delta)$-equivalent.

Let $I$ be the number of inequivalent components of $N(p)$. Then Proposition 5.1 implies that $I<\infty$.

Lemma 5.3. $N_{I}^{*}(\Delta)=N(p)$. 
Proof. Writing $N(p)=\Sigma g_{j} G_{\Delta}$, we need only show that $g_{j} \in N_{I}^{*}(\Delta)$. We note by Theorem 4.3 and Corollary 3.2 that $g_{j}(\Delta) \in \mathcal{L}(\Delta)$. Let $\left\{\sigma_{m}\right\}_{m=1}^{n}$ be the linkage of separators of nest type from $\Delta$ to $g_{j}(\Delta)$. If $n \leqslant I$, then $g_{j}(\Delta) \in \mathcal{L}_{I}(\Delta)$. Then (b) implies that $\Delta$ and $g_{j}(\Delta)$ are $N_{I}^{*}(\Delta)$-equivalent, therefore $g_{j} \in\left\langle G_{\Delta}, N_{I}^{*}(\Delta)\right\rangle=N_{I}^{*}(\Delta)$. Hence we assume that $n>I$. As an induction hypothesis we let $g_{j} \in N_{I}^{*}(\Delta)$ whenever the length of the linkage from $\Delta$ to $g_{j}(\Delta)$ is shorter than $n$. Let $\left\{\Delta_{1}, \ldots, \Delta_{n-1}\right\}$ be the subset of $\mathcal{L}(\Delta)$ such that $\sigma_{m}=\partial \Delta_{m-1} \cap \partial \Delta_{m}(m=1, \ldots, n)$, where $\Delta_{0}=\Delta$ and $\Delta_{n}=g_{j}(\Delta)$. Consider the finite sequence $\left\{\Delta, \Delta_{1}, \ldots, \Delta_{I}\right\}$, where $\left\{\Delta_{1}, \ldots, \Delta_{I}\right\}$ are the first $I$-terms of $\left\{\Delta_{1}, \ldots, \Delta_{n-1}\right\}$. Then $\left\{\Delta, \Delta_{1}, \ldots, \Delta_{I}\right\}$ is a sequence of length $I+1$ and consists of the elements of $E_{I}(\Delta)$. Hence it contains two $N_{I}^{*}(\Delta)$-equivalent components, say $\Delta_{k}$ and $\Delta_{l}, 0 \leqslant k<l \leqslant I$. Let $g$ be an element of $N_{I}^{*}(\Delta)$ such that $g\left(\Delta_{l}\right)=\Delta_{k}$ and $g\left(\sigma_{l+1}\right) \neq \sigma_{k}$. The last condition is satisfied if we multiply an element of $G_{\Delta_{k}}$ by $g$. Then $\left\{\sigma_{1}, \ldots, \sigma_{k}, g\left(\sigma_{l+1}\right), \ldots, g\left(\sigma_{n}\right)\right\}$ is the linkage of separators of nest type from $\Delta$ to $g\left(g_{j}(\Delta)\right)$ with length shorter than $n$. By our induction hypothesis, $g g_{j} \in N_{I}^{*}(\Delta)$ so $g_{j} \in N_{I}^{*}(\Delta)$. Q.E.D.

THEOREM 5.4. $N(p)$ is a nest group with $p$ as a residual limit point.

Proof. Lemma 5.3 and (a) imply that $N(p)$ is finitely generated. We show that each separator of $N(p)$ is of nest type. Assume there is a separator $\sigma$ of web type. Let $\Delta^{\prime}$ be the component of $N(p)$ such that $\sigma \subset \partial \Delta^{\prime}$. If $\Delta^{\prime} \in C(p)$, then $\Delta^{\prime}=c(q, p)$ for some $q \in L_{w}(G)$. Then $\sigma=\sigma(q, p)$. By Propositions 3.3 and 3.4 and Theorem $4.3, \sigma(q, p)$ is a separator of nest type of $N(p)$. This contradicts that $\sigma$ is of web type. Hence $\Delta^{\prime} \notin C(p)$ so $\Delta^{\prime} \in \mathcal{L}(\Delta)$. Let $D$ be the component of $\hat{\mathbf{C}} \backslash \boldsymbol{\sigma}$ not containing $\Delta^{\prime}$ and let $\Delta^{*}$ be a component of $N(p)$ contained in $D$. By Proposition 3.3 and Theorem 4.3 we may assume that $\Delta^{*} \in \mathcal{L}(\Delta)$. Then, since $\Delta^{\prime}$ lies outside $D$, the linkage of separators from $\Delta^{\prime}$ to $\Delta^{*}$ must contain $\sigma$. Hence there is an element $\Delta^{\prime \prime}$ of $\mathcal{L}(\Delta)$ such that $\sigma=\partial \Delta^{\prime} \cap \partial \Delta^{\prime \prime}$. This contradicts that $\sigma$ is of web type. Therefore we have shown that each separator of $N(p)$ is of nest type. Now, in view of Proposition 1.3 , to complete the proof of the theorem we need only show that $p$ is a residual limit point of $N(p)$. Proposition 2.1 and Theorem 3.5 imply that $p \in \Lambda(N(p))$. If there is a component $\Delta$ of $N(p)$ such that $p \in \partial \Delta$, then $\Delta$ is not a component of $G$ because we have chosen $p$ as a residual limit point of $G$. Theorem 4.3 implies that $\Delta$ is an element of $C(p)$ so $\Delta$ has the form $c(q, p)$. Then $\partial \Delta=\partial c(q, p)=\sigma(q, p)$. Since $\sigma(q, p)$ is a separator of $G, p \notin \sigma(q, p)=\partial \Delta$, a contradiction. Hence there is no component of $N(p)$ on whose boundary $p$ lies so $p$ is a residual limit point of $N(p)$. Q.E.D.

6. Minimal sets of generators. A set of generators of a finitely generated group is called a minimal set of generators if the number of its elements is minimum in the set of all the sets of generators. In [6] the following is shown.

THEOREM 6.1 [6]. Let $G$ be a finitely generated Kleinian group with $\Lambda_{0}(G) \neq \varnothing$. If $L_{1}(G) \neq \varnothing$, then there is a minimal set of generators $S_{1}$ of $G$ such that each element of $S_{1}$ is loxodromic and has the fixed points on $L_{1}(G)$.

Under our new classification of the residual limit points we refine Theorem 6.1 as follows. 
THEOREM 6.2. Let $G$ be a finitely generated Kleinian group with $\Lambda_{0}(G) \neq \varnothing$. If $G$ is neither a web group nor a nest group, then there is a minimal set of generators $S_{g}$ of $G$ such that each element of $S_{g}$ is loxodromic and has fixed points on $L_{g}(G)$.

Proof. Since $G$ is not a web group, by Proposition $1.3 G$ satisfies the assumption of Theorem 6.1. Let $S_{1}=\left\{\gamma_{1}, \ldots, \gamma_{n}\right\}$ be a minimal set of generators in Theorem 6.1. We shall first change $S_{1}$ into a minimal set of generators $S_{1}^{*}$ having the additional property that $S_{1}^{*}$ contains an element whose fixed points lie on $L_{g}(G)$. In order to do this we assume that the fixed points $\xi_{i}$ and $\xi_{i}^{\prime}$ of $\gamma_{i}$ lie on $L_{n}(G)(i=1, \ldots, n)$. Since $G$ is not a nest group, by Lemma 4.7 there are numbers $i$ and $j, 1 \leqslant i<j \leqslant n$, such that $N\left(\xi_{i}\right) \neq N\left(\xi_{j}\right)$. Then Proposition 2.2 and Theorem 3.5 imply that there is a separator of web type $\sigma$ separating $\xi_{i}$ from $\xi_{j}$. Denote by $D_{i}$ and $D_{j}$ the components of $\hat{\mathbf{C}} \backslash \sigma$ containing $\xi_{i}$ and $\xi_{j}$, respectively. Then by Proposition 2.2 and Lemma 4.7 $\xi_{i}^{\prime} \in D_{i}$ and $\xi_{j}^{\prime} \in D_{j}$. Since $D_{i}$ does not contain the fixed points of $\gamma_{j}$, and $\partial D_{i}=\sigma$ is a separator, $\gamma_{j}\left(D_{i}\right) \cap D_{i}=\varnothing$. Let $m$ be a large integer such that both isometric circles of $\gamma_{i}^{m}$ and $\gamma_{i}^{-m}$ lie in $D_{i}$. Then $\gamma_{j} \gamma_{i}^{m}$ maps the exterior of the isometric circle of $\gamma_{i}^{m}$ to the interior of the $\gamma_{j}$-image of the isometric circle of $\gamma_{i}^{-m}$. Since the $\gamma_{j}$-image of the isometric circle of $\gamma_{i}^{-m}$ lies outside $D_{i}, \gamma_{j} \gamma_{i}^{m}$ is a loxodromic element whose repelling and attractive fixed points lie in $D_{i}$ and $D_{j}$, respectively. Hence the fixed points of $\gamma_{j} \gamma_{i}^{m}$ are separated by $\sigma$ so they lie on $L_{g}(G)$. Replacing $\gamma_{j}$ by $\gamma_{j} \gamma_{i}^{m}$, we have $S_{1}^{*}$.

Next we shall change $S_{1}^{*}$ into $S_{g}$. Let $S_{1}^{*}=\left\{\gamma_{1}^{*}, \ldots, \gamma_{n}^{*}\right\}$ and let $\gamma_{1}^{*}$ be the element having fixed points on $L_{g}(G)$. Assuming $\gamma_{i}^{*}$ has fixed points on $L_{n}(G)$, we change it by one having fixed points on $L_{g}(G)$ as follows. Let $p$ and $q$ be the repelling and the attractive fixed points of $\gamma_{1}^{*}$, respectively, and let $\sigma$ be a separator of web type separating $p$ from $q$. Denote by $D_{p}$ and $D_{q}$ the components of $\hat{\mathbf{C}} \backslash \sigma$ containing $p$ and $q$, respectively. We choose a positive integer $m$ so large that $\gamma_{i}^{*}\left(\gamma_{1}^{*}\right)^{m}\left(D_{q}\right) \cap \gamma_{1}^{-m}\left(D_{p}\right)$ $=\varnothing$. This is possible because $\gamma_{i}^{*}(p) \neq q$. Then $\gamma_{i}^{*}\left(\gamma_{1}^{*}\right)^{2 m}$ is loxodromic with the repelling and the attractive fixed points in $\left(\gamma_{1}^{*}\right)^{-m}\left(D_{p}\right)$ and $\gamma_{i}^{*}\left(\gamma_{1}^{*}\right)^{m}\left(D_{q}\right)$, respectively. Since $\left(\gamma_{1}^{*}\right)^{-m}(\sigma)=\partial\left(\gamma_{1}^{*}\right)^{-m}\left(D_{p}\right)$ is a separator of web type separating the fixed points of $\gamma_{i}^{*}\left(\gamma_{1}^{*}\right)^{2 m}$, the fixed points of $\gamma_{i}^{*}\left(\gamma_{1}^{*}\right)^{2 m}$ lie on $L_{g}(G)$. We replace $\gamma_{i}^{*}$ by $\gamma_{i}^{*}\left(\gamma_{1}^{*}\right)^{2 m}$. Making a finite number of these operations, we reach $S_{g}$. Q.E.D.

\section{REFERENCES}

1. W. Abikoff, The residual limit sets of Kleinian groups, Acta Math. 130 (1973), 127-144.

2. W. Abikoff and B. Maskit, Geometric decompositions of Kleinian groups, Amer. J. Math. 99 (1977), 687-697.

3. T. Kuroda, S. Mori and H. Takahashi, Remarks on web groups, Ann. of Math. Studies, No. 97 , Princeton Univ. Press, Princeton, N. J., 1981, pp. 367-375.

4. B. Maskit, Intersections of component subgroups of Kleinian groups, Ann. of Math. Studies, No. 79, Princeton Univ. Press, Princeton, N. J., 1974, pp. 349-367.

5. T. Sasaki, On common boundary points of more than two components of a finitely generated Kleinian group, Tôhoku Math. J. 29 (1977), 427-437.

6. The residual limit sets and the generators of finitely generated Kleinian groups, Osaka J. Math. 15 (1978), 263-282.

7. $35-48$

Department of Mathematics, Faculty of Education, Yamagata University, Yamagata, JaPan 